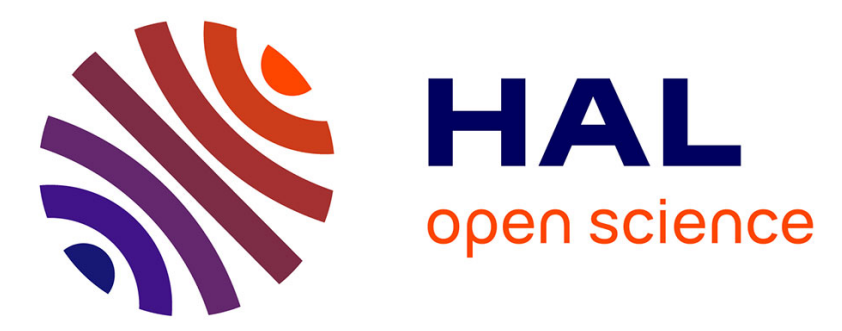

\title{
SUR LES CRITĖRES DE LOCALISATION DE LA DÉFORMATION PLASTIQUE DE TRACTION SIMPLE
}

\author{
C. Fressengeas, A. Molinari
}

\section{- To cite this version:}

C. Fressengeas, A. Molinari. SUR LES CRITÈRES DE LOCALISATION DE LA DÉFORMATION PLASTIQUE DE TRACTION SIMPLE. Journal de Physique Colloques, 1985, 46 (C5), pp.C5-121C5-125. 10.1051/jphyscol:1985515 . jpa-00224745

\section{HAL Id: jpa-00224745 https://hal.science/jpa-00224745}

Submitted on 1 Jan 1985

HAL is a multi-disciplinary open access archive for the deposit and dissemination of scientific research documents, whether they are published or not. The documents may come from teaching and research institutions in France or abroad, or from public or private research centers.
L'archive ouverte pluridisciplinaire HAL, est destinée au dépôt et à la diffusion de documents scientifiques de niveau recherche, publiés ou non, émanant des établissements d'enseignement et de recherche français ou étrangers, des laboratoires publics ou privés. 


\title{
SUR LES CRITĖRES DE LOCALISATION DE LA DÉFORMATION PLASTIQUE DE
}

\section{TRACTION SIMPLE}

\author{
C. Fressengeas et A. Molinari \\ L.P.M.M., GRECO "Grandes Déformations et Endommagement", Ile du Saulcy, \\ 57045 Metz Cedex, France
}

Résumé - L'instabilité de traction simple (striction) est décrite pour un matériau thermo-viscoplastique, conducteur de la chaleur, dans le cadre d'un schéma unidimènsionnel. Une méthode de perturbations relative permet de tenir compte de l'aspect instationnaire de l'écoulement, et de fournir des critères de localisation. On discute l'influence des paramètres mécaniques du matériau : écrouissage, sensibilité à la vitesse de déformation et à la température, conduction thermique, ainsi que des conditions aux frontières sur la localisation de l'écoulement plastique.

\begin{abstract}
Localization of plastic flow in tension (necking) in a thermal viscoplastic heat-conducting material is described within the framework of a unixial theory. A relative perturbation method is used, which allows to account for non-steadiness of plastic flow, and provides localization criteria. Effects of material parameters : strain hardening, strain rate sensitivity and thermal sensitivity, heat conduction, as well as the influence of boundary conditions on necking are discussed.
\end{abstract}

\section{I - INTRODUCTION}

Parmi les méthodes souvent utilisées pour décrire la localisation de l'écoulement plastique, figurent les techniques classiques de linéarisation $(1,7)$. Dans le cas d'une barre en traction simple, elles fournissent une bonne approximation de la déformation critique de début de localisation lorsque le matériau présente un comportement indépendant de la vitesse de déformation, et si le processus de déformation est quasi-statique : les effets de l'écrouissage et les effets thermiques sur l'initiation de la striction sont alors correctement décrits. En revanche, lorsque le matériau est sensible à la vitesse de déformation (8), ou lors d'un processus dynamique, un délai considérable à la striction peut intervenir $(6,7)$. Comme les méthodes lînéarisées fournissent seulement le début de la localisation, elles apparaissent incapables d'estimer les temps critiques de rupture, ou les valeurs limites de ductilité.

En outre, les analyses linéarisées classiques ne sont pas en mesure de fournir des critères de localisation. Ceux-ci n'ont été obtenus que dans un nombre restreint de cas particuliers, par exemple à force constante lorsque le matériau n'est pas conducteur de la chaleur, au moyen d'analyses non linéaires exactes (7).

L'objet du travail présenté est de fournir des critères de localisation plus étendus, prenant en considération la conduction thermique ainsi que des conditions aux limites variées. Ce résultat est obtenu grâce à l'introduction d'une méthode de perturbation relative linéarisée (voir aussi (9) dans ce volume, dans le contexte des bandes de cisaillement) ; cette méthode prend en compte l'aspect non stationnaire de l'écoulement plastique homogène. 
L'étude est basée sur un modèle unidimensionnel de la traction simple, contenant une non-uniformité initiale des variables; elle est donc limitée à l'approximation classique des grandes longueurs d'ondes, et l'aspect tridimensionnel de la distribution des contraintes n'est pas pris en considération.

Les équations fondamentales du problème sont tout d'abord formulées, et les résultats obtenus précédemment (7) résumés. Puis la méthode de perturbation relative est présentée ; enfin, certains résultats nouveaux sont brièvement évoqués. Une présentation plus détaillée et plus complète est fournie dans la référence (13).

\section{II - FORMULATION DU PROBLEME}

Considérons une barre axisymmétrique, de longueur et de section initiales lo et so, soumise à une sollicitation de traction simple; le matériau est supposé incompressible, et de masse volumique $\rho$. Dans un premier temps, une loi de comportement très simple est adoptée ; nous supposerons que :

$$
\tilde{\sigma}=\mu \theta^{\nu} \stackrel{m}{\dot{E}^{m}} \varepsilon^{n}
$$

où $\tilde{\sigma}, \tilde{\theta}, \tilde{\dot{\varepsilon}}$ et $\varepsilon$ désignent respectivement la contrainte vraie, la température, la vitesse de déformation et la déformation naturelles. $\mu, \nu$, m et $n$ sont des constantes empiriques ; $\nu, m$ et $n$ caractérisent respectivement la sensibilité à la température, à la vitesse $(m>0)$ et l'écrouissage.

Les équations qui gouvernent le problème sont, avec (2.1) :

$$
\begin{aligned}
& \rho \frac{\partial \tilde{V}}{\partial \tilde{t}}=\frac{\partial}{\partial \tilde{X}}\left(\tilde{\sigma} \mathrm{e}^{-\varepsilon}\right) \\
& \rho \frac{\partial \varepsilon}{\partial \widetilde{\mathrm{t}}}=\dot{\bar{\varepsilon}}=\frac{\partial \tilde{V}}{\partial \widetilde{X}} \cdot \mathrm{e}^{-\varepsilon} \\
& \frac{\partial \widetilde{G}}{\partial \widetilde{t}}=k\left(\frac{\partial^{2} \widetilde{\Theta}}{\partial \widetilde{x}^{2}}-\frac{\partial \widetilde{\Theta}}{\partial \widetilde{x}} \cdot \frac{\partial \varepsilon}{\partial \widetilde{x}}\right)+\beta \sigma \dot{\varepsilon} \\
& \frac{\partial \tilde{x}}{\partial \tilde{t}}=\tilde{v}
\end{aligned}
$$

$\tilde{t}$ et $\tilde{v}$ sont respectivement le temps et lạ vitesse de la section de coordonnée de Lagrange $\widetilde{X}$, et de coordonnée d'Euler $\widetilde{x}$.

$c$, $K$ et $\beta$ désignent respectivement la capacité thermique volumique, la conduction thermique, et la proportion de l'énergie de déformation plastique convertie en chaleur (coefficient de Taylor-Quinney) ; ces paramètres sont considérés comme constants dans cette étude. Les effets de conduction dûs à la variation de section : - $K \frac{\partial \widetilde{\Theta}}{\partial \widetilde{x}} \frac{\partial \widetilde{E}}{\partial \widetilde{x}}$ sont très petits dans l'approximation des grandes longueurs d'onde, et pourraient être négligés comme en (7); ils disparaîtront dans une analyse linéarisée. Notons en outre que $(\tilde{v}, \varepsilon, \widetilde{\sigma})$ sont des fonctions de $(\tilde{X}, t)$, de sorte que leurs dérivées partielles par rapport au temps sont aussi leurs dérivées matérielles. Les frontières sont soumises aux conditions (2.6.1) ou (2.6.2):

$$
\widetilde{v}(0, \tilde{t})=0, \quad \widetilde{v}\left(1_{0}, \widetilde{t}\right)=V, \tilde{t} \geqq 0
$$

( $V$ désigne la vitesse de l'extrémité mobile de la barre; $V$ peut être constante, ou pilotée de manière à obtenir une vitesse de déformation constante) 


$$
\tilde{v}(0, \tilde{t})=0, \quad \tilde{F}\left(1_{0}, \tilde{t}\right)=F_{0}, \tilde{t} \geqq 0
$$

F est la force constante appliquée à l'extrémité de la barre. La surface lătérale de la barre est supposée adiabatique, ainsi que les extrémités de la barre, pour lesquelles :

$$
\frac{\partial \widetilde{\theta}}{\partial \tilde{X}}(0, \tilde{t})=\frac{\partial \widetilde{\theta}}{\partial \widetilde{x}}\left(l_{0}, \tilde{t}\right)=0, \quad \tilde{\mathrm{t}} \geqq 0
$$

Lorsque les variables adimensionnelles :

$$
\begin{aligned}
& \mathrm{t}=\overline{\dot{\varepsilon}}_{\mathrm{o}} \tilde{\mathrm{t}} ; \quad \dot{\varepsilon}=\dot{\varepsilon}_{\bar{\varepsilon}} / \overline{\dot{\varepsilon}}_{\mathrm{o}} \\
& x=\tilde{x} / 1_{0} \quad ; \theta=\tilde{\theta} / \bar{\theta}_{0} \\
& \mathrm{X}=\tilde{\mathrm{x}} / 1_{0} \quad ; \sigma=\tilde{\sigma} / \bar{\sigma}_{\mathrm{o}} \\
& v=\tilde{v} / v_{0} \quad ; \quad F=\tilde{F} / F_{0}
\end{aligned}
$$

sont introduites, où $\bar{\theta}_{0}$ et $\overline{\dot{\varepsilon}}_{0}$ désignent respectivement les valeurs uniformes initiales de la température et de la vitesse de déformation, reliées à $\sigma_{0}$, $F_{0}$ et $v_{0}$ (la vitesse initiale de l'extrémité mobile) par :

$$
\bar{\sigma}_{0}=\mu \bar{\theta}_{0} \nu \overline{\dot{\varepsilon}}_{0}^{m} \quad ; \quad \bar{\varepsilon}_{0}=v_{0} / 1_{0} \quad ; \quad F_{o} / s_{0}=\bar{\sigma}_{0}
$$

les équations fordamentales se réduisent à :

$$
\begin{aligned}
& \frac{\partial \dot{\varepsilon}}{\partial t}=\frac{e^{-\varepsilon}}{\rho_{0}} \frac{\partial^{2}}{\partial X^{2}}\left(\Theta^{\nu} \dot{\varepsilon}^{m} \varepsilon^{n} e^{-\varepsilon}\right)-\dot{\varepsilon}^{2} \\
& \frac{\partial \varepsilon}{\partial t}=\dot{\varepsilon} \\
& \frac{\partial \theta}{\partial t}=k\left(\frac{\partial^{2} \theta}{\partial X^{2}}-2 \frac{\partial \theta}{\partial X} \frac{\partial \varepsilon}{\partial X}\right) e^{-2 \varepsilon}+\alpha \Theta^{\nu} \varepsilon^{n} \dot{\varepsilon}^{m+1}
\end{aligned}
$$
$\sigma, \nu, x$ ont été éliminés; $\rho_{o}, k$ et $\alpha$ sont des paramètres adimensionnels, $\rho_{o}$ est
significatif des effets d'inertie :

$$
\rho_{0}=\rho v_{0}^{2} / \dot{\sigma}_{0}
$$

$k$ est la conductivité thermique réduite :

$$
k=k / \mathrm{Cl}_{0}{ }^{2} \dot{\bar{\varepsilon}}_{\mathrm{o}}
$$

$\alpha$ contrôle le terme source de chaleur :

$$
\alpha=\beta \bar{\sigma}_{0} / c \bar{\theta}_{0}
$$

Les conditions aux limites réduites sont :

$$
\begin{aligned}
& v(0, t)=0, \quad v(1, t)=1, \quad t \geqq 0 \\
& \text { ou } v(0, t)=0, \quad \dot{\varepsilon}(1, t)=1, \quad t \geqq 0 \\
& \begin{array}{l}
\text { ou } \\
v(0, t)=0, \quad, \quad F(1, t)=1,
\end{array}
\end{aligned}
$$




$$
\frac{\partial \Theta}{\partial X}(0, t)=\frac{\partial \Theta}{\partial X}(1, t)=0 \quad, \quad t \geqq 0
$$

Pour les conditions initiales homogènes :

$$
\dot{\varepsilon}(X, 0)=1, \varepsilon(X, 0)=0, \theta(X, 0)=1,0 \leqq X \leqq 1
$$

les équations $(2.10 ; 2.12)$ admettent une solution homogène exacte ou approchée selon les conditions aux limites adoptées (13): $\left(\dot{\varepsilon}_{0}, \varepsilon_{0}, \theta_{0}\right)$. D'une manière classique, la stabilité d'une solution homogène est testée en recherchant une solution inhomogène de $(2.10 ; 2.12)$ de la forme :

$$
\begin{aligned}
& \dot{\varepsilon}(X, t)=\dot{\varepsilon}_{0}(t)+\delta \dot{\varepsilon}(t) \exp (i \xi X) \\
& \varepsilon(X, t)=\varepsilon_{0}(t)+\delta \varepsilon(t) \exp (i \xi X) \\
& \theta(X, t)=\theta_{0}(t)+\delta \theta(t) \exp (i \xi X)
\end{aligned}
$$

où les incréments $(\delta \dot{\varepsilon}, \delta \varepsilon, \delta \theta)$ sont supposés petits devant $\left(\dot{\varepsilon}_{0}, \varepsilon_{0}, \theta_{0}\right) . \xi$ est le nombre d'ondes de la perturbation. La substitution de $(2.18)$ dans $(2.10 ; 2.12)$ et la sélection des termes du premier ordre en $\left(\delta \varepsilon^{*}, \delta \varepsilon, \delta \theta\right)$ conduit à un système: $\frac{d X}{d t}=A(t) X d^{\prime}$ 'équations différentielles ordinaires dont les coefficients dépendent du temps. A titre d'exemple, l'analyse de l'équation caractéristique : $\operatorname{det}(A-\eta I)=0$ fournit, dans le cas d'un matériau non conducteur, peu sensible à la vitesse de déformation, en chargement quasi-statique, à la condition d'instabilité (7) :

$$
\varepsilon_{0} \frac{n}{1-\alpha \sigma_{0} / \theta_{0}}
$$

qui s'est révélée être en très bon accord avec les données expérimentales présentées par Ferron (5), en raison de la faible sensibilité à la vitesse de déformation de l'acier inoxydable utilisé. Cependant la croissance des perturbations (2.18) doit être beaucoup plus forte que celle de la déformation homogène pour valider ce critère, car la solution homogène doit être stationnaire dans la théorie linéarisée classique. Nous présentons maintenant une méthode de perturbation tenant davantage compte de l'instabilité de la déformation homogène.

\section{III - ANALYSE DE PERTURBATIONS LINEARISEES RELATIVES}

Définissons les variables relatives :

$$
\lambda=\varepsilon / \varepsilon_{0}, \psi=\varepsilon / \varepsilon_{0}, \varphi=\theta / \theta_{0}
$$

La substitution de (3.1) dans les équations réduites $(2.10 ; 2.12)$ fournit les équations :

$$
\begin{aligned}
& \frac{\partial \lambda}{\partial t}=\frac{1}{\rho_{0}} e^{-\psi \varepsilon_{0}} \frac{\sigma_{0}}{\varepsilon_{0}} \frac{\partial^{2}}{x^{2}}\left(\varphi^{\nu} \lambda^{m} \psi^{n} \mathrm{e}^{-\psi \varepsilon_{0}}\right)-\varepsilon_{0}\left(\lambda^{2}-\lambda\right) \\
& \frac{\partial \psi}{\partial t}=\frac{\dot{\varepsilon}_{0}}{\varepsilon_{0}}(\lambda-\psi) \\
& \frac{\partial \varphi}{\partial t}=k\left(\frac{\partial^{2} \varphi}{\partial X^{2}}-2 \varepsilon_{0} \frac{\partial \varphi}{\partial X} \frac{\partial \varepsilon}{\partial X}\right) e^{-2 \psi \varepsilon_{0}}+\frac{\dot{\theta}_{0}}{\Theta_{0}}\left(\varphi^{\nu} \psi^{n} \lambda^{m+1}-\varphi\right)
\end{aligned}
$$


Il est clair que la solution homogène de ces équations est :

$$
\lambda_{0}=1, \phi_{0}=1, \varphi_{0}=1
$$

et qu'elle ne dépend plus du temps. Nous cherchons maintenant une solution inhomogène de la forme :

$$
\begin{aligned}
& \lambda(y, t)=1+\delta \lambda(t) \exp (i \xi x) \\
& \psi(y, t)=1+\delta \psi(t) \exp (i \xi x) \\
& \varphi(y, t)=1+\delta \varphi(t) \exp (i \xi x)
\end{aligned}
$$

L'examen de la croissance du défaut relatif $(\delta \lambda, \delta \psi, \delta \varphi)$ conduit à des conclusions relatives à la localisation, alors que la croissance du défaut absolu est représentative de 1 'instabilité $(11,12,13)$. La linéarisation des équations $(3.2 ; 3.4)$ conduit à un système différentiel linéaire $: \frac{d X}{d t}=B(t) . X$ dont les coefficients dépendent du temps ; l'analyse de ce système au moyen des résultats de Coddington-Levinson (10) montre que pour des matériaux tels que : $\nu<1$ la localisation de l'écoulement ne peut être évitée, pour aucune des conditions aux limites : $(2.15 .1 ; 2.15 .2 ; 2.15 .3)$. Pour des matériaux thermo-durcissants tels que : $\nu>1$, les résultats concernant la localisation dépendent au contraire des conditions aux limites, ainsi que de la sensibilité à la vitesse de déformation (13).

\section{REMERCIEMENTS}

Les auteurs remercient la D.R.E.T. pour le support financier fourni dans le cadre du contrat $83 / 1071$.

\section{REFERENCES}

/ 1/ Hart, E.w. , Acta Metall., 15, 351 (1967).

/ 2 / Campbell, J.D., J. Mech. Phys. solids, 15, 359 (1967).

/ 3/ Jonas, J.J., Holt, R.A. and Coleman C.E., Acta Metall. 24, 911 (1976).

/ 4 / Argon, A.S., the Inhomogeneity of Plastif Flow, Chapter 7, American Society of Metals, Metals Park Ohio (1973).

/ 5/ Ferron, G., Mater. Sci. Engng 49, 241 (1981).

/ 6/ Taylor, J.W., Harlow, F.H. and Amsden, A.A., J. Appl. Mech., 45, 105 (1978).

/ $7 /$ Fressengeas, C. and Molinari, A., Acta Metall., 33, 387 (1985).

/ 8 / Hutchinson, J.W. and Neale, K.W., Acta Metall. 25, 831 (1977).

/ $9 /$ Fressengeas, C. and Molinari, A., A Relative Stability Analysis for Shear Band Formation, Dymat Paris (1985).

$/ 10 /$ Coddington, E.A. and Levinson, N., Theory of Ordinary Differential Equations, Mac Graw Hill, New York (1955).

/11/ Molinari, A., Seme Colloque Mécanique et Métallurgie de Tarbes, ADISTA/ATS pp $1-43$ (1984).

/12/ Molinari, A., J.M.T.A. to be published (1985).

$113 /$ C. Fressengeas ; A. Molinari, to be published. 